\title{
Improving the Usable Capacity of Ad Hoc Networks
}

\author{
Christian Maihöfer, Tim Leinmüller, and Reinhold Eberhardt \\ DaimlerChrysler AG, Research Vehicle IT and Services, \\ P.O. Box 2360, 89013 Ulm, Germany, \\ \{Christian.Maihoefer|Tim.Leinmueller|Reinhold.Eberhardt\} \\ @DaimlerChrysler.com
}

\begin{abstract}
Recent research has shown that multi-hop ad hoc networks suffer from a low capacity and moreover that an individual node's capacity for long-distance communication decreases the more nodes are participating in the ad hoc network. The usable capacity of ad hoc networks is further decreased by the forwarding metric of usual routing protocols, which try to minimize the number of hops or try to minimize the remaining distance to the receiver. This results in traffic concentration at one or several areas in the network, what we will refer to as network center(s), and thus in a decreased usable capacity. Especially in the automotive domain, where road infrastructure and road traffic flow frequently create network centers, solving this issue is of particular interest.

In this paper we will show that the network center is indeed a bottleneck for communication which limits a network's capacity. Then we will present a new forwarding approach, which relieves the network center from handling an excessive part of the traffic. Instead of just trying to minimize the distance to the receiver, this approach additionally tries to avoid routing through the network center. Simulation results show, that the usable capacity of the ad hoc network is increased with this approach by approximately $30 \%$.
\end{abstract}

\section{Introduction}

Ad hoc networks are wireless networks of nodes which may be mobile and may work without infrastructure. Such networks provide a convenient, a cheap, and for some scenarios a robust way for communication. That is why ad hoc networks like Bluetooth or IEEE 802.11, which provide both an access point mode and an ad hoc mode, receive more and more attention.

Recently, ad hoc networks are even considered for long distance communication, with senders and receivers outside each others direct wireless communication range. These ad hoc networks are called multi-hop ad hoc networks. This means, some or all nodes of the ad hoc network provide routing functionality (see [1] for an overview of ad hoc routing protocols).

Due to the growing space of large ad hoc networks, which allows spatial reuse of the wireless medium, the total capacity of the network grows. Unfortunately, 
this is not true for long-distance communication in multi-hop ad hoc networks, as they require cooperation of many nodes. Essentially the broadcast nature of the wireless transmission affects many nodes. All neighboring nodes, i.e. nodes in direct wireless communication range to the initial sender or forwarding node receive the transmission and must not simultaneously transmit themselves. This is a serious problem for multi-hop ad hoc networks, since it limits an individual node's capacity for long-distance communication to a value that decreases the more nodes are joining the ad hoc network.

Although the limited capacity is a serious problem, there are several ways to relax this problem somewhat, i.e. to increase the usable capacity. First of all, if traffic patterns show a high degree of locality between sender and receiver, i.e. if only a small degree of traffic has to be routed over several hops, the usable capacity can be significantly higher and sufficient for many purposes. Another option would be to use the ad hoc network only as an access network and use a wired backbone network to transmit long-distance traffic. However, in this paper we do not want to make such assumptions. Instead we try to increase the usable capacity for long-distance communication inside ad hoc networks by eliminating a serious bottleneck, the area of traffic concentration, i.e. the network center.

In the automotive domain, e.g. in communication scenarios as they have been investigated in the FleetNet project [2, network centers are determined by road networks and road traffic flow. Consequently, network centers are located in areas with high road density and high road traffic flows, e.g. road traffic concentration points in urban areas, whereas the network border lies in direction to areas with lower road traffic flow, for instance rural areas. Therefore we assume network centers can be determined by all nodes by using cartographic data from the navigation system and radio broadcasts on traffic conditions.

The network center is a serious bottleneck particularly for the limited capacity of ad hoc networks in two ways. First of all, most long-distance traffic crosses the network center, since routing protocols often try to minimize the number of hops or try to minimize the remaining distance to the receiver. This leads to an increased traffic concentration at the center of the network which becomes a bottleneck. Second, for long-distance traffic, the usable capacity of the network is limited by the capacity of the center. If the center has a lower capacity than the remaining network, all long-distance traffic suffer from the low center capacity. Note that such a situation is not just a theoretical problem, rather it is quite likely that the capacity of the center is lower than the capacity of other parts of the network. For example, if the node density in the center is above the average node density of the network, instead of increasing the capacity of the center this reduces its capacity.

In this paper we address the problem of the ad hoc network center as a bottleneck which decreases usable capacity. After discussing some related work in the following section, we will start with presenting simulations to validate that such a problem indeed exists in Section 3 Then in Section 4 we present a new routing metric, which relives the center from forwarding most long-distance 
traffic. Simulation results show, that the usable capacity of the ad hoc network is increased with this approach. Finally, we will conclude with a brief summary.

\section{Related Work}

Protocols for routing in ad hoc networks can be classified into topology-based and position-based or geographic approaches 3. Topology-based schemes use only information about existing neighborhood links rather than additional physical (geographical) position information of the participating nodes. Topologybased approaches can be further divided into table-driven and source-initiated on-demand driven protocols [1. Basically, table driven protocols attempt to maintain consistent and up-to-date routing information among all nodes, while source-initiated on-demand driven protocols create routes only when necessary to deliver a packet. Therefore, the former approach is also known as a proactive routing approach and the latter as a reactive approach.

For geographical routing protocols, three basic forwarding strategies can be identified: 1) greedy forwarding, 2) restricted directional flooding, and 3) hierarchical forwarding. With greedy forwarding a node forwards a packet to a neighbor that is located closer to the destination. If this forwarding strategy fails, since there may be situations in which there is no closer node to the destination than the forwarding node, recovery strategies have to deal with it. Restricted directional flooding is similar to the greedy approach with the modification that a packet is forwarded to some neighbors rather than to just one neighbor. Finally, hierarchical forwarding tries to improve scalability by forming a hierarchy of non-equal nodes. In our simulations in the following sections we will use a greedy routing algorithm.

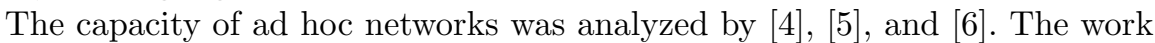
in 4 has first analyzed the capacity of ad hoc networks and has shown that the capacity per node in an ad hoc network is $\Theta(1 / n \log (n))$, where $n$ is the total number of nodes. This is a quite pessimistic result, since it indicates that large ad hoc networks are not feasible. However, the authors also pointed out that under certain conditions communication with nearby neighbors will be able with constant bit rate independent of the network size.

In 5 the analytical work is extended to reflect the influence of different traffic patterns on scalability of the per node capacity. The authors also determined the influence of forwarding algorithms and 802.11 MAC interaction which resulted in an estimation of missing constants from the previous work of 4 .

In 6 the analysis is extended to consider the mobility of nodes. They have shown that long term per node capacity can stay constant. Their presented algorithm makes use of the known result that local communication with constant bit rate is possible. The basic approach is to distribute a packet to many different nodes, which store the packets. Now the nodes' movements will finally bring one of the relaying nodes close to the intended destination of the packet. The packet is then forwarded from the relaying node to the destination node. Therefore, only a constant number of hops is required independent of the network size, thus the 


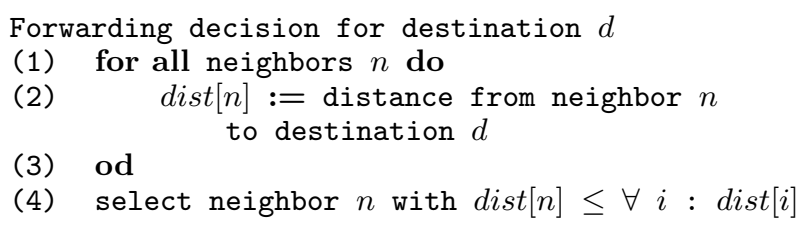

Fig. 1. Basic forwarding algorithm

per node capacity stays constant. However, their assumptions will rather not apply to most ad hoc networks and furthermore, packet delivery delays may become infinite.

\section{The Network Center Bottleneck}

From the results of related work we know that the capacity of ad hoc networks is a critical resource and that we should try to use it very economically. For multi-hop long-distance traffic a particular bottleneck that prevents to use the full theoretical capacity is the center of the network, through which most traffic is routed and which therefore limits the usable capacity. Our first goal is to prove this assumption.

\subsection{A Simple Counting Model}

In Figure 1 a basic forwarding scheme is shown which is used in the following as the reference approach. This scheme is similar to a greedy forwarding algorithm in position aware networks. For non-position aware forwarding protocols, the used distance metric in the figure is replaced by another metric, e.g. the hop distance to the destination. Although we will assume a position-aware greedy algorithm in this paper, the observations and results will apply for many other protocols, too.

When we apply this basic forwarding scheme to a simple network model with random traffic we can count the number of received packets per node in order to detect a hot spot. This is shown in Figure 2 $\mathrm{b}$ with a regular flat network consisting of 10 nodes for each dimension, resulting in 100 nodes in total. It is assumed, that a packet transmission can be received by exactly 8 neighbors, for nodes on the network border accordingly less neighbors (see Figure 2a). The traffic consists of 2.000 message transmissions between randomly chosen nodes.

The results in Figure 2 b show that there is a traffic concentration at the center of the network, which limits the overall usable capacity of the network and degrades throughput. If we change the system model, e.g. if we assume that a node has only 4 neighbors instead of 8 , or if we count the number of forwarded packets per node instead of the number of received packets, the absolute values change but the qualitative results do not. 

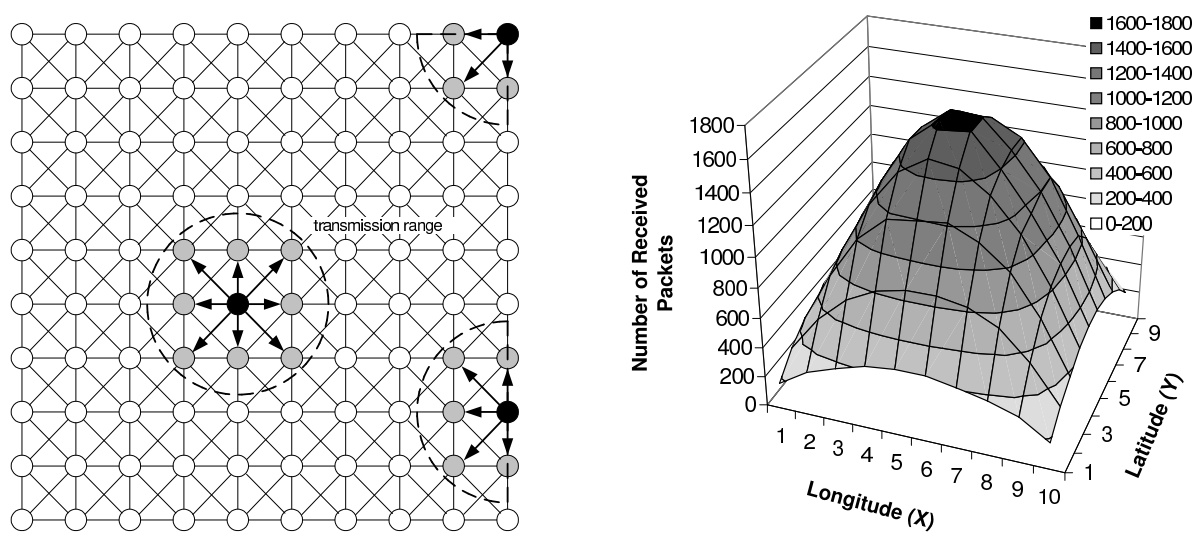

Fig. 2. a) Network model and b) load per node with random traffic

\subsection{Simulations of the Network Center Bottleneck}

Besides this rather simple approach of analyzing the network load, we have also made extensive simulation studies, which allow deeper insight. With the network simulator NS-2 7 and the CMU wireless extensions, we were able to run simulations in a quite realistic wireless network scenario. The simulated IEEE 802.11 network was configured to have a $250 \mathrm{~m}$ transmission range. It uses the RTS/CTS scheme preceding every data packet exchange and ACKs to confirm successful packet reception. The network was static, i.e. no node movement was considered. We have used a position-based greedy routing algorithm, which was derived from GPSR 8 but lacks the perimeter mode, i.e. no backtracking mechanism is in place if a packet transmission reaches a dead end. In such a case the packet cannot be delivered and is dropped. As we assumed that the position of nodes are known to all other nodes no location service was necessary.

Figure 3 a shows the received link layer packets per node for a similar scenario like Figure 2 b. A regular network of 10x10 nodes and latitudinal and longitudinal distance of $100 \mathrm{~m}$ between nodes was simulated. The traffic consisted of 2000 message transmissions between random senders and receivers. The figure shows the contour lines from a top to bottom view of the three dimensional graph, which allows better readability. As it can be seen, the qualitative result is identical to the simple counting model, there is a traffic concentration at the center. The quantitative figures are somewhat higher, the center node has to process 2412 packets in the simulation compared to 1673 packets from the numerical approach. This is caused by the higher wireless transmission range, which is $250 \mathrm{~m}$ in the simulation, which means that 12 neighbors are within reach of a wireless transmission, while in the numerical approach 8 neighbors are within reach. A decreased transmission range of $150 \mathrm{~m}$ in the simulation leads to 8 neighbors within transmission range and a maximum of 1617 received packets for the center, which is quite close to the numerical results. 

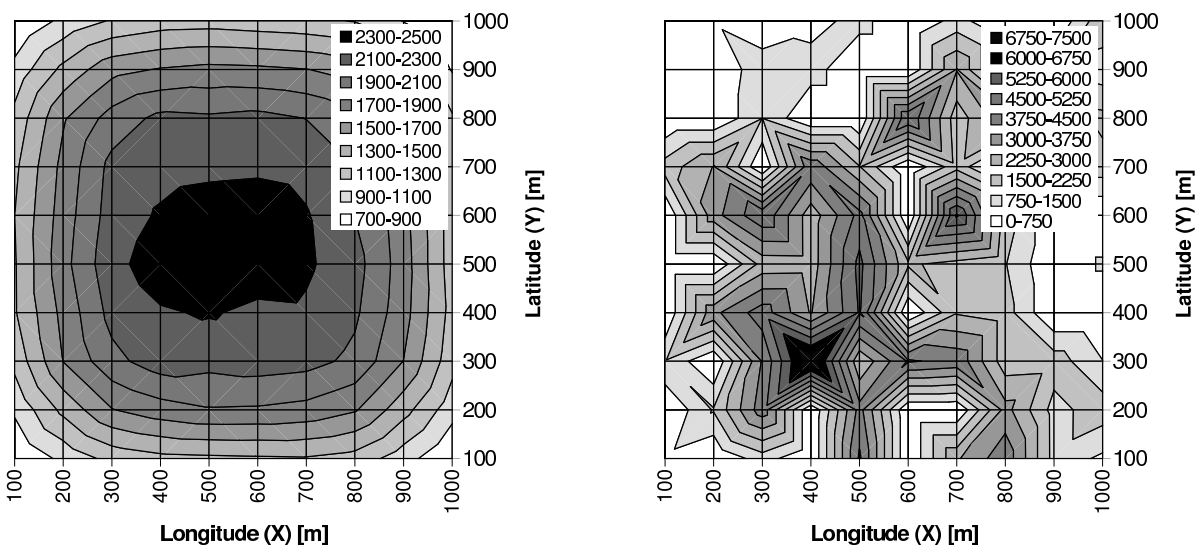

Fig. 3. Network load per node a) regular network and b) network with randomly placed nodes

Figure $3 \mathrm{~b}$ shows the results for a non-regular network, consisting of 100 randomly placed nodes in a $1000 \times 1000 \mathrm{~m}^{2}$ area. Although it is more difficult to see a center hot spot, since there are gaps in the network where no node is located, the traffic concentration at the edges is obviously less than the traffic concentration in and around the center.

\subsection{Simulations of the Center Density Impact}

Now we will prove our second claim from Section 1 that the center, besides being a traffic bottleneck, is moreover likely to have a lower capacity than other parts of the network. A higher node density decreases the capacity of an ad hoc network, since more neighbors are involved in each forwarding step, which is likely for the center of a network.

Figure 4 shows the simulation results for different densities of the center region compared to the average density of a $10 \times 10$ network in a grid of $1000 \times 1000 \mathrm{~m}^{2}$ with $250 \mathrm{~m}$ wireless transmission range. A density below $100 \%$ means that the density of the center is lower than the density of other parts of the network, accordingly a density above $100 \%$ means that the center density is above average. All 2000 message transmissions were initiated at simulation start (simulation time 0s). Figure 4 a depicts the number of transport layer messages received at the destination at simulation time 1s. For example, with density $100 \%$, from the 2000 transmissions only 77 messages have arrived at the destination, which means that the network is highly congested. In such a congested scenario, the network is at its capacity limit. As a consequence, the measured number of arrived messages corresponds with the usable network capacity. If the center density is increased, the usable network capacity decreases. It increases, if the center density is below the average node density. 

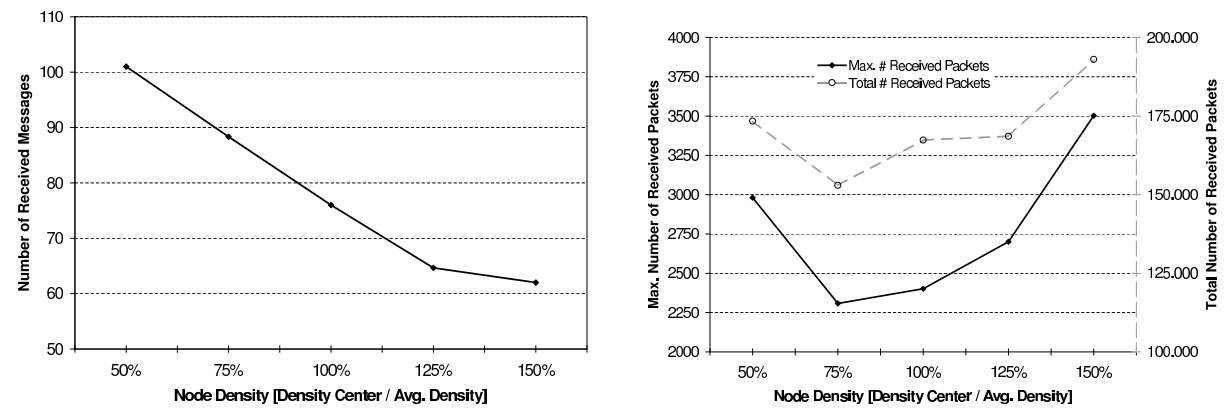

Fig. 4. Network throughput and network load

Figure $4 \mathrm{~b}$ shows the the maximum number of received packets at link layer and the aggregated total number of received packets. Both show the expected result that if the node density of the center is above average the number of received packets increase. But they also show that if the node density of the center is below average, the number of received packets increase also. In this case the edges of the network have a node density above normal and hence they cause an increased number of packets. However, as the edges of the network have to forward only a traffic share below average, the network capacity does not decrease, instead it even increases since the center is relieved.

From these results we can conclude that the usable capacity of an ad hoc network is limited by the capacity of the network center. In the following section we will present how the usable network capacity can be increased by a modified forwarding scheme that relieves the center from forwarding load.

\section{Increasing the Usable Capacity of Ad Hoc Networks}

As the network center limits the usable capacity of an ad hoc network, our goal is to relieve the center from forwarding too many long-distance transmissions.

\subsection{A Center-Relieving Forwarding Scheme}

The basic forwarding scheme, which was shown in Figure 1 considers only one metric to optimize the forwarding process, the distance to the destination. We modify this forwarding scheme by a second metric, the distance to the center of the network. While we try to minimize with each forwarding step the distance to the destination, we also try to maximize the distance to the network center, which accomplishes that the center is bothered with less traffic. Note that there is surly a trade-off between both metrics.

In Figure 5 we show in detail our modified forwarding scheme. In line (7) it is ensured that each forwarding step makes progress towards the destination. However, if there are several neighbors which are closer to the destination, the 
Forwarding decision for destination $d$

(1) for all neighbors $n$ do

(2) distdest $[n]:=$ distance from neighbor $n$

to destination $d$

(3) distcent $[n]:=$ distance from neighbor $n$

to the network center

(4)

(5) od

forwardmetric $[n]:=$ distdest $[n]$

- WeightFactor $*$ distcent $[n]$

(6) currdist $:=$ current distance to destination $d$

(7) select neighbor $n$ with distdest $[n]<$ currdist and forwardmetric $[n] \leq \forall i:$ forwardmetric $[i]$

Fig. 5. Forwarding algorithm that relives the network center

decision which neighbor is selected depends on both, the progress towards the destination and the distance to the network center. The configuration parameter WeightFactor allows to adjust to which extent the network center is to be relieved. If the parameter is set equal to 0 , then the distance to the center is not considered and the result of the forwarding scheme is equal to the result of the basic forwarding scheme from Figure 1. On the other hand, if the parameter is set to a value larger than 1, then it is more important to get away from the center than coming closer to the destination. This is not a reasonable forwarding decision, since now too much load is shifted from the center to the borders of the network, which makes the borders a possible bottleneck.

An example for the center-relieving forwarding mechanism is given in Figure 6. a. While the regular forwarding mechanism routes a packet through the network center, the center-relieving mechanism avoids the network center. Figure 6. b shows the contour lines of a NS-2 simulation with the scenario introduced in Section 3.2 i.e. a regular 10 x 10 network with 2000 message transmissions. The WeightFactor was set equal to 0.7. Besides resulting in a lower maximum number of received packets compared with Figure 3 a, it also shows that the algorithm is effective in relieving the network center.

\subsection{Evaluation of the Usable Capacity Gain}

Coming back to our initial goal, improving the usable capacity of an ad hoc network, we evaluate with simulations if and to what extent the center-relieving forwarding can extend the usable network capacity.

We used again the NS-2 simulation environment for IEEE 802.11 wireless LANs introduced in the previous sections. Figure 7 shows the results for a simulation run of $25 \mathrm{~s}$ and a $2000 \times 2000 \mathrm{~m}^{2}$ network consisting of 500 nodes. Between simulation time 1 and 20s, 5000 message transmissions were initiated between randomly selected senders and receivers. Figure 7 a shows the absolute number of delivered messages with respect to the simulation time. The solid line depicts 

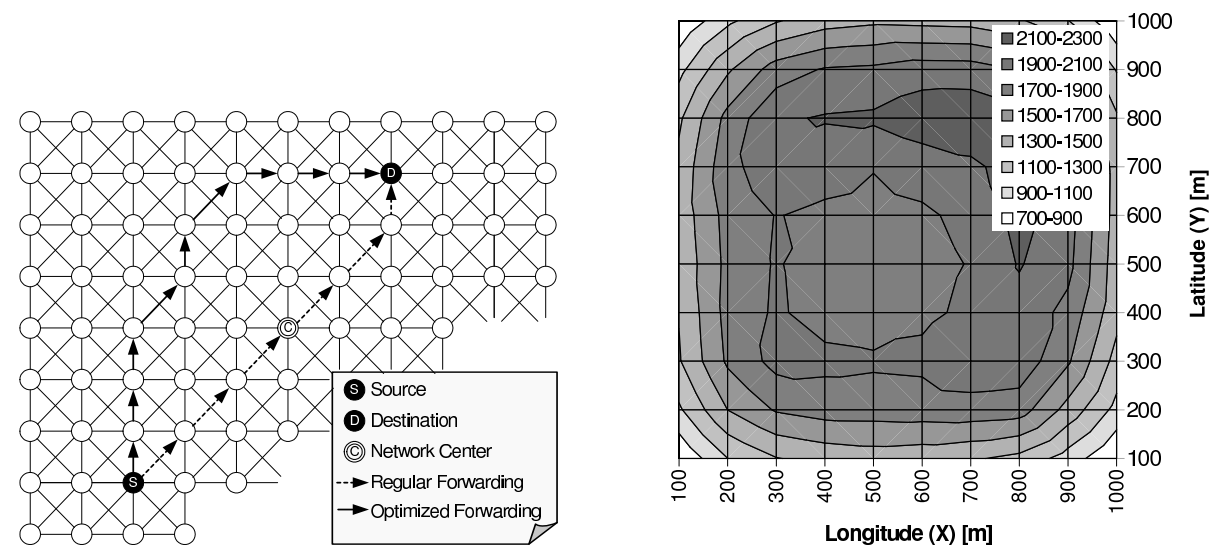

Fig. 6. a) Example for greedy and center-relieving forwarding and b) Effect of center-relieving forwarding on the network load distribution
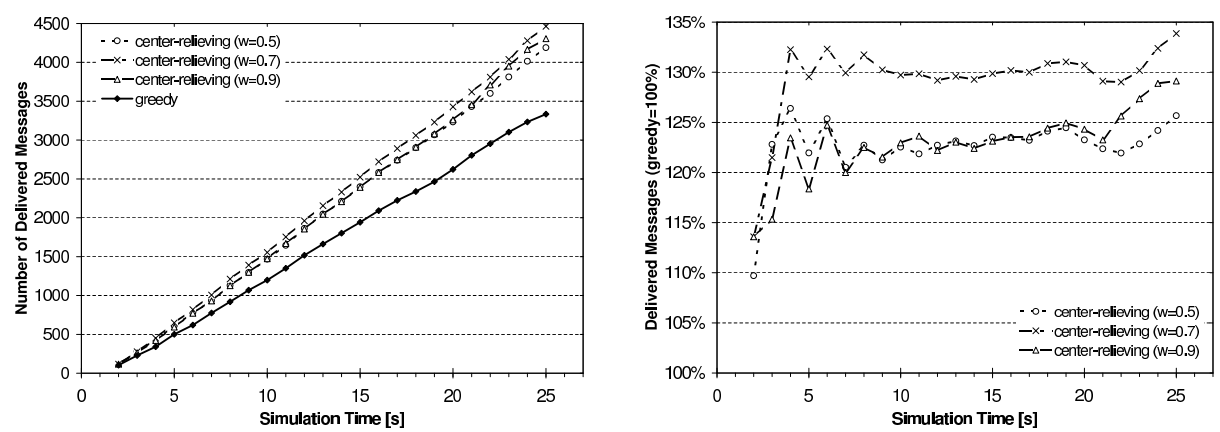

Fig. 7. Capacity comparison of greedy routing and center-relieving routing with respect to simulation time 

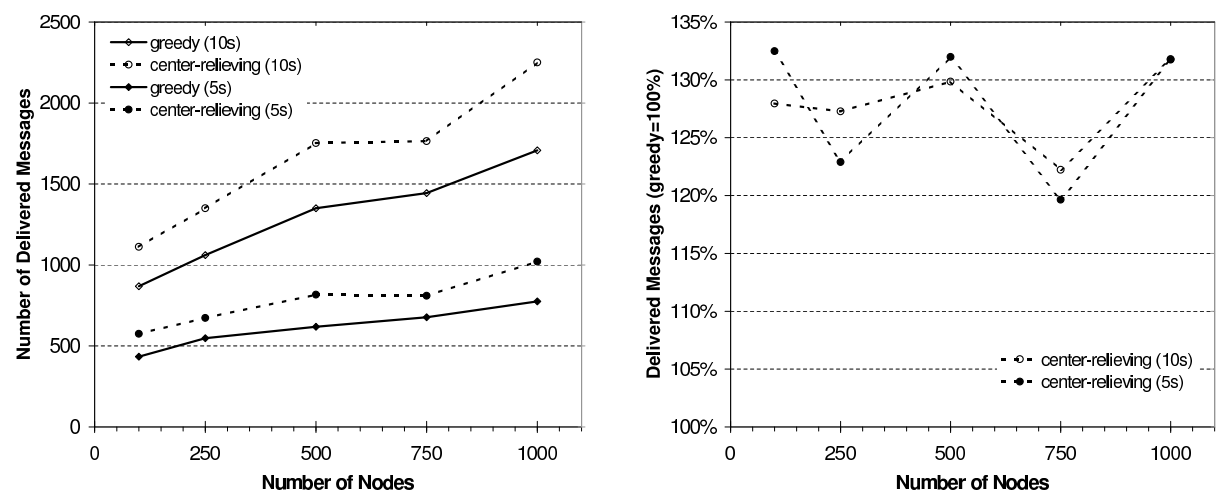

Fig. 8. Capacity comparison of greedy routing and center-relieving routing with respect to network size

the results for greedy routing. With greedy routing, only 3334 messages out of 5000 can be delivered during the $25 \mathrm{~s}$ simulation time, because the network center is overwhelmed with packet forwarding.

The center-relieving algorithm can achieve an increased number of delivered messages. The results for center-relieving routing depend on the WeightFactor, which was set to $0.5,0.7$, and 0.9 , respectively. We can see that if the WeightFactor equals 0.7 , with 4463 delivered messages out of 5000 the best results are achieved in this scenario.

In Figure 7. b the relative improvement in delivered messages compared to greedy routing is depicted, which allows a better comparison. After an initial increase at simulation start, center-relieving routing is able to achieve a $30 \%$ higher delivery rate than greedy routing, which stays roughly constant during the simulation time. Note that this throughput improvement is paid with slightly longer paths from sender to receiver, i.e. increased number of hops and therefore an increased total number of received packets at all nodes in the network. We have measured this increased total number of received packets at all nodes to be approximately $10 \%$ in our simulations. Compared to the $30 \%$ throughput gain, a $10 \%$ increased overall load seems tolerable.

A further comparison of greedy routing with center-relieving routing is shown in Figure 8, with different network sizes of 100, 250, 500, 750 and 1000 nodes. The nodes are randomly placed in a grid of $1000 \times 1000 \mathrm{~m}^{2}, 1500 \times 1500 \mathrm{~m}^{2}, 2000 \times 2000 \mathrm{~m}^{2}$, $2500 \times 2500 \mathrm{~m}^{2}$ and $3000 \times 3000 \mathrm{~m}^{2}$, respectively. Again, 5000 message transmissions are initiated between randomly chosen senders and receivers during the time interval of 1 and 20s. The total simulation time was 25s. Center-relieving routing was configured with a WeightFactor of 0.7 . The graphs show the results of the received messages in a $5 \mathrm{~s}$ and a $10 \mathrm{~s}$ interval after simulation start.

Figure 8 a shows the absolute results, while Figure 8 b shows the relative improvement compared to greedy routing. The increasing number of delivered 
messages results from the constant number of initiated message transmissions. With more nodes in the network, every one has to process less traffic. More interesting is the comparison between greedy routing and center-relieving routing. The simulation results show that the usable capacity respectively throughput gain of center-relieving routing is in the range of $20 \%-33 \%$, with different network sizes. Although the observed results are not constant, which results from the random generation of traffic in the simulation, there is no indication that with smaller or larger networks the achieved gain in capacity changes fundamentally. In summary, we have shown that center-relieving routing can increase the usable capacity of ad hoc networks by approximately up to $30 \%$, which is independent of the network size as well as simulation time.

\section{Conclusion}

Multi-hop ad hoc networks suffer from a low capacity and an individual node's capacity for long-distance communication decreases the more nodes are participating in the ad hoc network. In this paper we have shown an approach to increase the usable capacity for long-distance communication inside ad hoc networks by eliminating a serious bottleneck, the area of traffic concentration, i.e. the network center. Usually, routing protocols try to minimize the number of hops or try to minimize the remaining distance to the receiver, which results in a traffic concentration at the network center, limiting the overall network capacity.

We have presented a new forwarding approach for ad hoc networks, which relieves the center from handling most long-distance traffic. Instead of just trying to minimize the distance to the receiver, our approach additionally tries to avoid routing through the network center. Simulation results show, that the usable capacity of the ad hoc network is increased with this approach by approximately $30 \%$.

In further work we will evaluate the influence of the WeightFactor, which determines to what extent the network center is to be relieved from forwarding traffic, in more detail. Also we will include node mobility in our scenarios. Furthermore, we plan to elaborate the results of our work in urban area automotive communication scenarios. On the other hand, we need to investigate mechanisms to determine the network center, without relying on cartographic information.

\section{References}

1. E. Royer and C. Toh, "A review of current routing protocols for ad-hoc mobile wireless networks," IEEE Personal Communications, vol. 6, no. 2, pp. 46-55, Apr. 1999.

2. W. Franz, R. Eberhardt, and T. Luckenbach, "Fleetnet - Internet on the road," Eighth World Congress on Intelligent Transportation Systems, Sydney, Australia, 2001. 
3. Martin Mauve, Jörg Widmer, and Hannes Hartenstein, "A survey on position-based routing in mobile ad hoc networks," IEEE Network, vol. 15, no. 6, pp. 30-39, Dec. 2001.

4. P. Gupta and P. R. Kumar, "The capacity of wireless networks," IEEE Transactions on Information Theory, vol. 46, no. 2, pp. 388-404, Mar. 2000.

5. Jinyang Li, Charles Blake, Douglas S. J. DeCouto, Hu Imm Lee, and Robert Morris, "Capacity of ad hoc wireless networks," in Proceedings of the ACM/IEEE International Conference on Mobile Computing and Networking (MobiCom). Oct. 2001?, ACM Press.

6. M. Grossglauser and D. Tse, "Mobility increases the capacity of ad-hoc wireless networks," IEEE/ACM Transactions on Networking, vol. 10, no. 4, Aug. 2002.

7. S. Bajaj, L. Breslau, D. Estrin, K. Fall, S. Floyd, M. Handley P. Haldar, A. Helmy, J. Heidemann, P. Huang, S. Kumar, S. McCanne, R. Rejaie, P. Sharma, K. Varadhan, Y. Xu, H. Yu, and D. Zappala, "Improving simulation for network research," Technical Report 99-702, University of Southern California, USA, 1999.

8. B. Karp and H.T. Kung, "Greedy perimeter stateless routing for wireless networks," in Proceedings of the Sixth ACM/IEEE International Conference on Mobile Computing and Networking (MobiCom 2000), Boston, USA, Aug. 2000, pp. 243-254. 